\title{
Prospects for SUSY dark matter after the LHC Run 1
}

\section{Emanuele Bagnaschi*}

Member of the MasterCode collaboration ${ }^{\dagger}$

DESY, Notkestraße 85, D-22607 Hamburg, Germany

E-mail: emanuele.bagnaschiddesy.de

In this proceeding we present the results of the recent phenomenological study by the MasterCode collaboration [1] of the Dark Matter (DM) annihilation mechanisms in the currently allowed parameter space of the CMSSM, NUHM1, NUHM2 and pMSSM10 models. We have assumed that the lightest neutralino $\chi_{1}^{0}$ is the Lightest Supersymmetric Particle (LSP). The annihilation mechanisms involved in these models range from coannihilation with the next-toLSP (NLSP) particle (e.g. the lightest stau $\tilde{\tau}_{1}$, stop $\tilde{t}_{1}$ or chargino $\tilde{\chi}_{1}^{ \pm}$) to resonant annihilation via s-channel exchange of a Higgs $(\mathrm{h} / \mathrm{H} / \mathrm{A})$ boson or a $\mathrm{Z}$ boson and enhanced annihilation rate in the so-called focus-point region due to a larger Higgsino component of the LSP. Special attention has been given to the interplay of future LHC searches and direct DM detection experiments in the exploration of the allowed regions. We have found that the $\tau_{1}$ coannihilation regions of the CMSSM, NUHM1 and NUHM2 will be largely covered by future LHC searches for $E_{T}^{\prime}$ signatures and long-lived charged particles, while direct detection DM experiments (LZ) will probe the H/A funnel, focus point and $\tilde{\chi}_{1}^{ \pm}$coannihilation regions. In the case of the pMSSM10, LHC experiments will be able to explore only parts of the preferred $68 \%$ CL region, where $\tilde{\chi}_{1}^{ \pm}$is the dominant mechanism. On the other hand, DM direct detection experiments will be able to probe the favored pMSSM10 parameter space more extensively.

The European Physical Society Conference on High Energy Physics

22-29 July 2015

Vienna, Austria

\footnotetext{
* Speaker.

${ }^{\dagger}$ http://mastercode.web.cern.ch/
} 


\section{Introduction}

The goal of the MasterCode collaboration is to provide global fits for SUSY models, accounting for the most accurate theoretical predictions and for the latest experimental searches. Among the experimental constraints, one of the most relevant is the the Cold Dark Matter (CDM) relic density. Indeed the latter is now very precisely determined by measurements of the cosmic microwave background, which yield $\Omega_{\mathrm{CDM}} h^{2}=0.1186 \pm 0.0020$ [2]. This constraint was already included in a strings of recent analyses of different MSSM models by the MasterCode collaboration $[3,4,5,6]$ and by some of its members independently [7]. In all cases it was assumed that R-parity is conserved and that the DM candidate is the lightest neutralino, $\chi_{1}^{0}$. While in these papers the importance of the CDM relic density in selecting the allowed parameter space was already stressed, a systematic study of the DM annihilation mechanisms was not performed. With our latest study [1], presented in this proceeding, we try to fill this gap. This will also allow us to stress the complementary importance of collider searches and of DM direct detection experiments.

Our analysis of the DM annihilation mechanisms is performed in all the four different MSSM models already mentioned above and that were studied by the collaboration. In detail, we consider:

- The constrained MSSM (CMSSM) [8, 9], where the soft SUSY-breaking parameters are universal and given at the GUT scale. Consequently, the model is fully determined by the value of the soft SUSY-breaking mass for the SUSY scalars $\left(m_{0}\right)$, by the value of the softSUSY breaking mass for for the SUSY fermions $\left(m_{1 / 2}\right)$ and by the scalar trilinear couplings $\left(A_{0}\right)$, at a common unification scale.

- The Non-Universal Higgs Mass (NUHM1) model [10], where the two soft SUSY-breaking masses for the Higgs doublets are allowed to be different from $m_{0}$, though fixed to the same value, $m_{H_{1}}^{2}=m_{H_{2}}^{2} \neq m_{0}$.

- The Non-Universal Higgs Mass (NUHM2) model [11], where the degeneracy of the softSUSY breaking Higgs mass terms is lifted, $m_{H_{1}}^{2} \neq m_{H_{2}}^{2} \neq m_{0}$.

- A version of the phenomenological MSSM (pMSSM10) [12], where we allow for 10 different independent parameters in the Lagrangian. The latter are the three gaugino soft SUSYbreaking masses $\left(M_{1}, M_{2}, M_{3}\right)$, two squark masses $\left(m_{\tilde{q}_{1,2}}, m_{\tilde{q}_{3}}\right)$, a common mass term for all the sleptons $\left(m_{\tilde{l}}\right)$, a common trilinear coupling $\left(A_{0}\right)$, the Higgs mixing parameter $(\mu)$, the pseudoscalar Higgs mass $\left(M_{A}\right)$ and the ratio of the vevs of the two Higgs doublets $\left(\tan \beta \equiv v_{2} / v_{1}\right)$. The equality of the soft SUSY-breaking masses of the two first generations of squarks is dictated by the absence of significant Flavor Changing Neutral Currents (FCNC) beyond the SM ones, while the assumptions for all sfermions that the left-handed mass is equal to the right-handed one is postulated only for simplicity. All the inputs are given at the electroweak scale, at difference with the GUT models where the parameters were defined at the GUT scale.

We present the results in a series of phenomenologically relevant two-dimensional projections of the multi-dimensional parameter space. In the same planes, we will also show the reach of forthcoming LHC searches and the sensitivity of future direct-detection DM experiments, where relevant. 


\section{The MasterCode framework}

All our analyses are performed using MasterCode, a frequentist global fitting framework for SUSY models. MasterCode is written in $\mathrm{C}++$, Python and Cython. It implements an efficient way of interfacing the different codes providing the theoretical predictions in the computation of the global $\chi^{2}$ function. The multi-dimensional parameter space is sampled using the MultiNest algorithm $[13,14,15]$. A detailed description of all the included experimental constraints and of the codes used for the theoretical predictions is included in ref. $[3,7,4,5,6]$.

\section{The DM annihilation mechanisms}

We use a list of pre-defined heuristic mass-degeneracy measures as a proxy to the importance of a specific DM annihilation mechanism. We have checked with micrOMEGAs [16] that indeed they describe correctly the DM phenomenology, yielding the dominant process (i.e. the mechanism that gives the largest contribution of final states, usually being $\gtrsim 50 \%$ ). In the case of the GUT models, they are:

$$
\begin{gathered}
\tilde{\tau}_{1} \text { coann. (pink): }\left(\frac{m_{\tilde{\tau}_{1}}}{m_{\tilde{\chi}_{1}^{0}}}-1\right)<0.15, \\
\tilde{\chi}_{1}^{ \pm} \text {coann. (green) : }\left(\frac{m_{\tilde{\chi}_{1}^{ \pm}}}{m_{\tilde{\chi}_{1}^{0}}}-1\right)<0.1, \\
\tilde{t}_{1} \text { coann. (grey) : }\left(\frac{m_{\tilde{t}_{1}}}{m_{\tilde{\chi}_{1}^{0}}}\right)-1<0.2, \\
\text { A/H funnel (blue) }: \quad\left|\frac{M_{A}}{m_{\tilde{\chi}_{1}^{0}}}-2\right|<0.4, \\
\text { focus point (cyan) : } \quad\left(\frac{\mu}{m_{\tilde{\chi}_{1}^{0}}}\right)-1<0.3 .
\end{gathered}
$$

For each mechanism we have also specified the corresponding color that will be used in the figures. In the case of the pMSSM10, we define to two additional measures:

$$
\begin{gathered}
h \text { funnel (magenta) }:\left|\frac{M_{h}}{m_{\tilde{\chi}_{1}^{0}}}-2\right|<0.4, \\
Z \text { funnel (orange) }:\left|\frac{M_{Z}}{m_{\tilde{\chi}_{1}^{0}}}-2\right|<0.4 .
\end{gathered}
$$

We also relax the numerical condition on $\tilde{\chi}_{1}^{ \pm}$coannihilation measure, re-defining it to $\mid m_{\tilde{\chi}_{1}^{ \pm}} / m_{\tilde{\chi}_{1}^{0}}-$ $1 \mid<0.25$, so that it more accurately reproduces the dominance of this mechanism in this model Notice that there is no focus-point behavior in the pMSSM10, however we still use the same notation and color to represent the regions where the LSP has an enhanced Higgsino component and annihilation rate. 


\section{The DM planes}

We now show the results of our analyses as projections in two-dimensional planes. For each fixed coordinates in the 2D-plane, the point in the full multi-dimensional parameter space with the lowest $\chi^{2}$ is selected. In all the figures we delimit with red and blue contours the area in the model planes where $\Delta \chi^{2}=2.30$ and $\Delta \chi^{2}=5.99$, respectively. These correspond roughly to the regions allowed at the $68 \% \mathrm{CL}$ and $95 \% \mathrm{CL}$. The best fit points are shown as filled green stars.

\subsection{The $\left(m_{0}, m_{1 / 2}\right)$ plane}

In fig. 1 we show the dominant annihilation mechanisms in the $\left(m_{0}, m_{1 / 2}\right)$ plane for the CMSSM (upper left), NUHM1 (upper right) and NUHM2 (bottom left) models. For the pMSSM10 (bottom right) we plot on the other hand the $\left(m_{\tilde{q}}, m_{\chi_{1}^{0}}\right)$ plane, since $m_{0}$ and $m_{1 / 2}$ have no meaning in this model. To understand the current and future reach of LHC searches, concerning the GUT models, we plot with a solid purple line the current 95\% CL exclusion from the SUSY $E_{T}$ searches [17], while we use a dashed purple contour for the $5 \sigma$ discovery reach for $E_{T}$ searches with $3000 \mathrm{fb}^{-1}$ [18] at $14 \mathrm{TeV}$. These correspond approximately to the $95 \% \mathrm{CL}$ exclusion reach with $300 \mathrm{fb}^{-1}$ at $14 \mathrm{TeV}$. In the case of the pMSSM10, the dashed (dash-dotted) contour corresponds to the $3000 \mathrm{fb}^{-1} 95 \% \mathrm{CL}$ exclusion sensitivity at $14 \mathrm{TeV}$, under the assumption that $m_{\tilde{g}} \gg m_{\tilde{q}}$ (with the hypothesis that $m_{\tilde{g}}=4.5 \mathrm{TeV}$ ).

From the upper left plot, we see four distinct areas in the CMSSM plane. In the low- $m_{0}$-low$m_{1 / 2}$ region, we see that $\tilde{\tau}_{1}$ coannihilation is the dominant DM annihilation mechanism. In this region we also find the best fit point. Still at low $m_{0}$ but at larger $m_{1 / 2}$, we observe an hybrid of $\tilde{\tau}_{1}$ coannihilation and $\mathrm{A} / \mathrm{H}$ funnel; for larger $m_{0}$ and $m_{1 / 2}$, the plane is dominated by the $\mathrm{A} / \mathrm{H}$ funnel. Finally, at large $m_{0}$ and at lower $m_{1 / 2}$, the focus point behavior is the relevant one.

We notice that the $\tilde{\tau}_{1}$ coannihilation region is very close to the current $95 \%$ CL exclusion limit from $E_{T}$ searches (solid purple curve) and it will be fully explored with $3000 \mathrm{fb}^{-1}$ of integrated luminosity (dashed purple curve). These high-luminosity searches will also probe part of the hybrid and a small section of the H/A funnel and focus point regions.

In the case of the NUHM1 (top right), we notice a $\tilde{\tau}_{1}$ coannihilation region similar to the CMSSM one, which borders now with a more extended hybrid region. The latter extends up to $m_{1 / 2} \sim 3500 \mathrm{GeV}$. We also note the appearance of a large area dominated by $\tilde{\chi}_{1}^{ \pm}$coannihilation, for $2500 \lesssim m_{1 / 2} \lesssim 4000 \mathrm{GeV}$ (besides a small hybrid-region peninsula) and spanning all the range in $m_{0}$. Here we have that $\mu \lesssim M_{1}$ and therefore $\tilde{\chi}_{1}^{0}, \tilde{\chi}_{2}^{0}$ and $\tilde{\chi}_{1}^{ \pm}$are nearly mass degenerates and the LSP has a mainly Higgsino component. Finally, for large $m_{0}$ and moderate value of $m_{1 / 2}, \mathrm{H} / \mathrm{A}$ funnel dominates. The current LHC searches border now both the $\tilde{\tau}_{1}$ coannihilation and $A / H$ funnel regions. Future searches will probe part of these two regions and of the hybrid area. However it will not be sensitive to the $\tilde{\chi}_{1}^{ \pm}$coannihilation region, where the best fit point is also located.

Concerning the NUHM2, we see that now, with respect to the NUHM1, the $\tilde{\tau}_{1}$ coannihilation region is much larger while the extensions of the hybrid region is reduced. We also notice that there is a small island around $\left(m_{0}, m_{1 / 2}\right) \sim(500,2000) \mathrm{GeV}$ that is dominated by $\tilde{t}_{1}$ coannihilation. The latter is close to the current $95 \%$ CL exclusion limits and it will be completely probed by the $3000 \mathrm{fb}^{-1} E_{T}$ searches, which will also test part of the $\tilde{\tau}_{1}$ coannihilation and A/H funnel regions. 

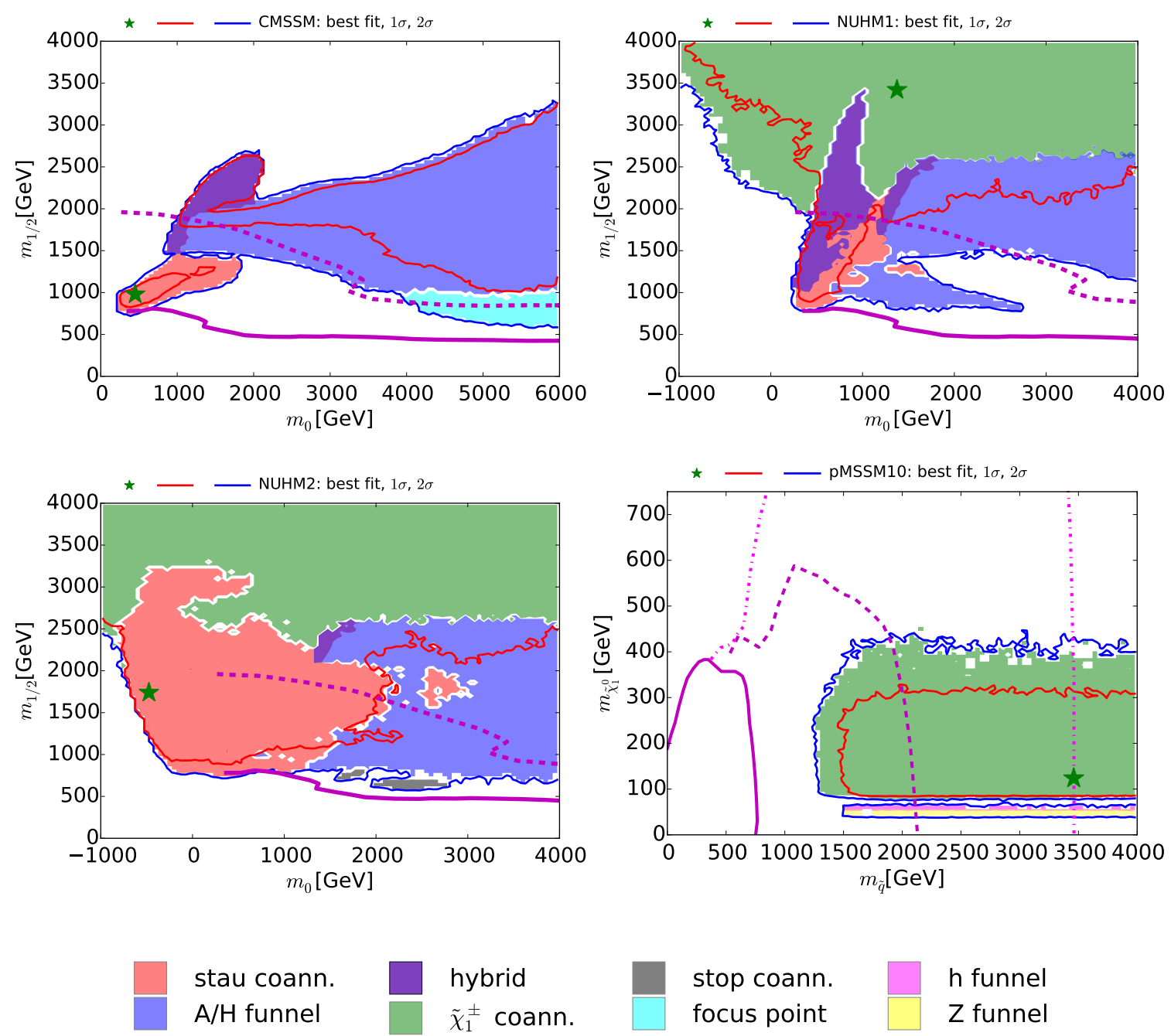

h funnel

Z funnel

Figure 1: The $\left(m_{0}, m_{1 / 2}\right)$ plane for the CMSSM (top left), NUHM1 (top right) and NUHM2 (bottom left). For the pMSSM10, we plot in, the bottom right panel, the $\left(m_{\tilde{q}}, m_{\tilde{\chi}_{1}^{0}}\right)$ plane. In the plots, the shadings represent the various DM annihilation mechanisms, with the used color coding reported in the legend. The $68 \% \mathrm{CL}(95 \% \mathrm{CL})$ is shown as a red (blue) contour. For the GUT models, the solid purple contours show the current LHC 95\% CL exclusions from $E_{T}^{\prime}$ searches while the dashed purple line $5 \sigma$ discovery reach with $3000 \mathrm{fb}^{-1}$ in the same channels, at $14 \mathrm{TeV}$. The latter corresponds roughly with the $95 \% \mathrm{CL}$ exclusion sensitivity with $300 \mathrm{fb}^{-1}$ at $14 \mathrm{TeV}$. In the case of the pMSSM10, the dashed line correspond to the $3000 \mathrm{fb}^{-1}$ 95\% CL exclusion sensitivity with the assumption that $m_{\tilde{g}} \gg m_{\tilde{q}}$, while the dash-dotted curve correspond to a fixed $m_{\tilde{g}}=4.5 \mathrm{TeV}$.

Finally, the pMSSM10 is characterized by the overwhelming dominance of the $\tilde{\chi}_{1}^{ \pm}$coannihilation mechanism. In this area the LSP is Bino-like. We also observe a band at lower $m_{\tilde{\chi}_{1}^{0}}$ where $h$ and $Z$ funnels assume the role of dominant DM annihilation mechanism. Future $E_{T}$ LHC searches will be able to test part of the allowed regions, as it can be seen from the purple contours, though the precise amount is dependent on the details of the SUSY spectrum. 

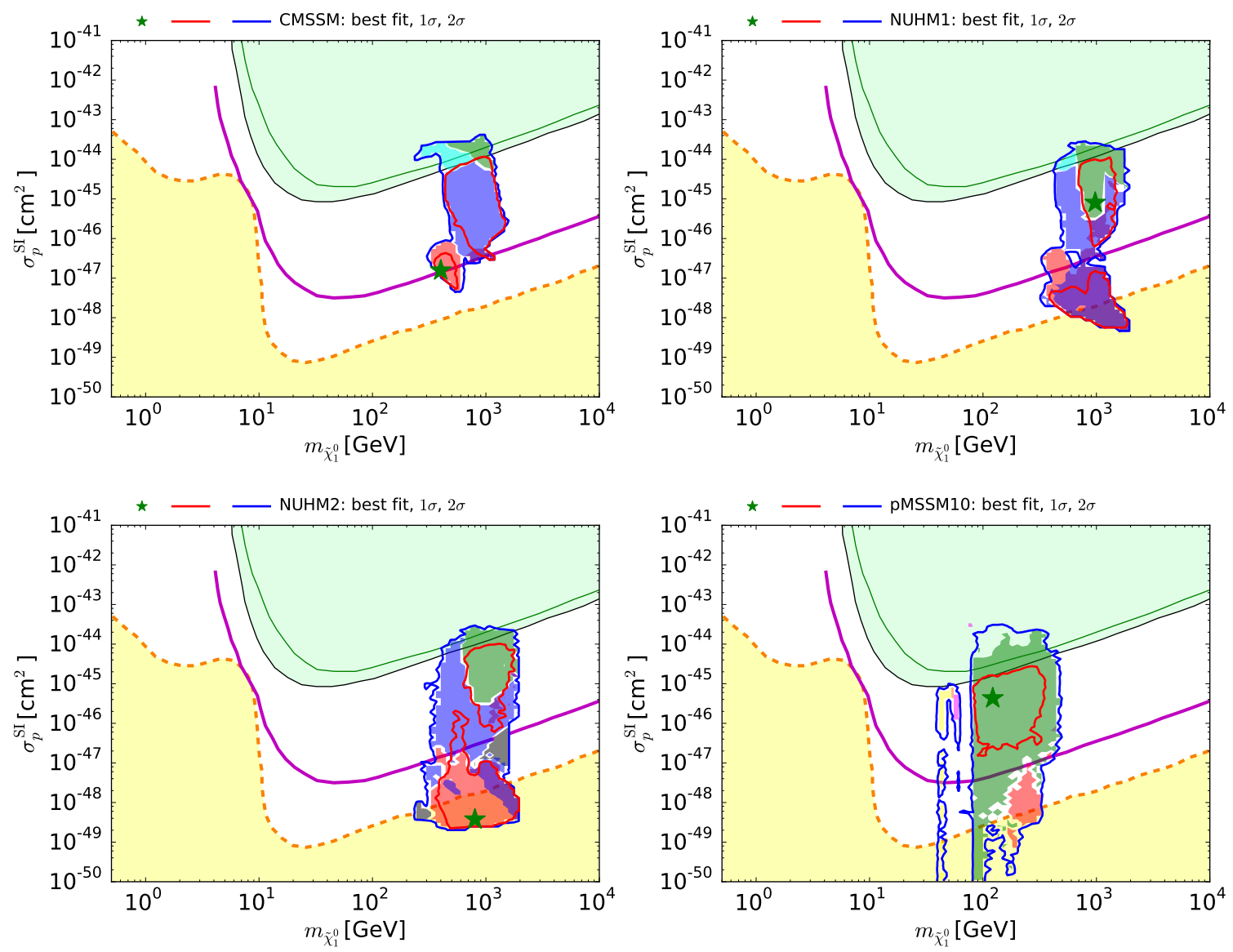

stau coann. A/H funnel

hybrid

$\tilde{\chi}_{1}^{ \pm}$coann. stop coann. focus point h funnel

Z funnel

Figure 2: The $\left(m_{\tilde{\chi}_{1}^{0}}, \sigma_{p}^{\mathrm{SI}}\right)$ plane for the CMSSM (top left), NUHM1 (top right) and NUHM2 (bottom left) and the pMSSM10 (bottom right). In the plots, the shadings represent the various DM annihilation mechanisms, with the corresponding color coding reported in the legend. The 68\% CL (95\% CL) is shown as a red (blue) contour. The current sensitivities of the XENON100 and LUX experiments are shown with a green and black lines respectively. In purple we plot the estimated exclusion reach of the LZ experiment at $95 \%$ CL. The orange dashed contour delimit the "neutrino floor", below which every DM signal will be overwhelmed by the background from astrophysical neutrinos.

\subsection{Direct DM searches}

In fig. 2 we show the $\left(m_{\tilde{\chi}_{1}^{0}}, \sigma_{p}^{\text {SI }}\right)$ planes ${ }^{1}$ for the four models under analysis: the CMSSM (top left), the NUHM1 (top right), the NUHM2 (bottom left) and the pMSSM10 (bottom right). The sensitivity of the current experiments are shown with a green line for XENON100 and with a black line for LUX. We also plot the 95\% CL exclusion reach of the future LUX-Zepelin (LZ) experiment with a purple line. The dashed orange contour is the so-called "neutrino floor", below which the

\footnotetext{
${ }^{1}$ The details of our computation of the spin-independent scattering cross-section on a proton are described in ref. [6].
} 
astrophysical neutrino background would overwhelm any genuine DM signal.

In the case of the CMSSM (top left), we see that the current experiments already probe quite deeply the focus point and the $\tilde{\chi}_{1}^{ \pm}$coannihilation region. We also notice that the LZ experiment will be able to test the remaining part of the $\tilde{\chi}_{1}^{ \pm}$coannihilation region and the H/A funnel region. It is interesting to note that, on the other hand, the $\tilde{\tau}_{1}$ coannihilation region is not completely accessible by current or planned direct detection DM experiments. However it will be fully explored by the LHC searches, as shown in figure 1.

In the NUHM1 we see a similar picture, with the $\tilde{\chi}_{1}^{ \pm}$coannihilation region and H/A region within the LZ reach and the $\tilde{\tau}_{1}$ coannihilation and hybrid regions reaching beyond and extending into the neutrino floor. However, as in the CMSSM, these parameter space regions will be at least partially covered by LHC searches.

Similar observations are also valid for the NUHM2, with the difference that the best fit point, which lies in the $\tilde{\tau}_{1}$ coannihilation region, is now below the neutrino floor. Also, the $\tilde{t}_{1}$ coannihilation region will be only marginally probed by LZ.

Finally, in the pMSSM10 we observe that the LZ experiment will be able to test a large part of the region dominated by $\tilde{\chi}_{1}^{ \pm}$coannihilation, completely exploring the section allowed at $68 \% \mathrm{CL}$ and therefore the best fit point. In this plot we show as "uncolored" the regions where none of the mechanisms we studied is dominant. They will be probed by LZ as well. The very small value of $\sigma_{p}^{S I}$ allowed in the pMSSM10 are due to cancellations between different contributions in the $\sigma_{p}^{S I}$ cross-section. It was show in ref. [6] that similar cancellations occur when spin-independent cross section on the neutron is considered.

\section{Conclusions and outlook}

In the study of the MasterCode collaboration presented in [1] and reported in this proceeding, we have studied the various DM annihilation mechanisms that play a dominant role in bringing the observed CDM relic density in the range measured by cosmological observations. The study has been performed for the CMSSM, the NUHM1, the NUHM2 and the pMSSM10 models.

In the case of the GUT models, we have found that usually between three and four mechanisms are dominant, depending on the region of the parameter space. These are $\tilde{\tau}_{1}, \tilde{t}_{1}, \tilde{\chi}_{1}^{ \pm}$coannihilations, $\mathrm{H} / \mathrm{A}$ funnels and the focus-point behavior. There are also hybrid regions where both $\tilde{\tau}_{1}$ coannihilation and H/A funnel play a role. On the other hand, in the pMSSM10, $\tilde{\chi}_{1}^{ \pm}$coannihilation dominates in almost all of the preferred parameter space.

Concerning GUT models, we have also observed that future $E_{T}$ LHC searches will be able to probe a significant part of the $\tilde{\tau}_{1}$ coannihilation region. In the NUHM2, the $\tilde{t}_{1}$ coannihilation region will be probed as well. On the other hand, underlying the complementarity between collider and direct-detection DM experiments, the $\tilde{\chi}_{1}^{ \pm}$coannihilation and the focus point regions could be completely explored by LZ. The latter will also be able to explore the H/A funnel region. Conversely, direct-detection DM experiments will not be able to study the $\tilde{\tau}_{1}$ coannihilation region as completely as it will possible via the LHC.

In the case of the pMSSM10, we see that significant regions of the $\tilde{\chi}_{1}^{ \pm}$coannihilation parameter space, including all the area preferred at $68 \% \mathrm{CL}$, will be probed both by the LHC and by the LZ experiments. 
While not reported here, in the original article we have also extensively studied other parameter planes, stressing different phenomenological aspects and the perspective of using other LHC searches to derive information on the dominant DM annihilation mechanism (assuming that the $\tilde{\chi}_{1}^{0}$ is the dominant CDM component). Moreover we have also pointed out the possibility of long-lived charged particles in the GUT models and therefore the opportunity of searching for signatures of metastable charged particles at the LHC.

\section{References}

[1] E. A. Bagnaschi et al., arXiv:1508.01173 [hep-ph].

[2] P. A. R. Ade et al. [Planck Collaboration], arXiv:1502.01589 [astro-ph.CO].

[3] O. Buchmueller et al., Eur. Phys. J. C 72 (2012) 2243 [arXiv:1207.7315 [hep-ph]].

[4] O. Buchmueller et al., Eur. Phys. J. C 74 (2014) 6, 2922 [arXiv:1312.5250 [hep-ph]].

[5] O. Buchmueller et al., Eur. Phys. J. C 74 (2014) 12, 3212 [arXiv:1408.4060 [hep-ph]].

[6] K. J. de Vries et al., Eur. Phys. J. C 75 (2015) 9, 422 [arXiv:1504.03260 [hep-ph]].

[7] M. Citron, J. Ellis, F. Luo, J. Marrouche, K. A. Olive and K. J. de Vries, Phys. Rev. D 87 (2013) 3, 036012 [arXiv:1212.2886 [hep-ph]].

[8] M. Drees and M. M. Nojiri, Phys. Rev. D 47 (1993) 376 [arXiv:hep-ph/9207234]; H. Baer and M. Brhlik, Phys. Rev. D 53 (1996) 597 [arXiv:hep-ph/9508321]; Phys. Rev. D 57 (1998) 567 [arXiv:hep-ph/9706509]; H. Baer, M. Brhlik, M. A. Diaz, J. Ferrandis, P. Mercadante, P. Quintana and X. Tata, Phys. Rev. D 63 (2001) 015007 [arXiv:hep-ph/0005027]; J. R. Ellis, T. Falk, G. Ganis, K. A. Olive and M. Srednicki, Phys. Lett. B 510 (2001) 236 [hep-ph/0102098].

[9] G. L. Kane, C. F. Kolda, L. Roszkowski and J. D. Wells, Phys. Rev. D 49 (1994) 6173 [arXiv:hep-ph/9312272]; J. R. Ellis, T. Falk, K. A. Olive and M. Schmitt, Phys. Lett. B 388 (1996) 97 [arXiv:hep-ph/9607292]; Phys. Lett. B 413 (1997) 355 [arXiv:hep-ph/9705444]; J. R. Ellis, T. Falk, G. Ganis, K. A. Olive and M. Schmitt, Phys. Rev. D 58 (1998) 095002 [arXiv:hep-ph/9801445]; V. D. Barger and C. Kao, Phys. Rev. D 57 (1998) 3131 [arXiv:hep-ph/9704403]; J. R. Ellis, T. Falk, G. Ganis and K. A. Olive, Phys. Rev. D 62 (2000) 075010 [arXiv:hep-ph/0004169]; L. Roszkowski, R. Ruiz de Austri and T. Nihei, JHEP 0108 (2001) 024 [arXiv:hep-ph/0106334]; A. Djouadi, M. Drees and J. L. Kneur, JHEP 0108 (2001) 055 [arXiv:hep-ph/0107316]; U. Chattopadhyay, A. Corsetti and P. Nath, Phys. Rev. D 66 (2002) 035003 [arXiv:hep-ph/0201001]; J. R. Ellis, K. A. Olive and Y. Santoso, New Jour. Phys. 4 (2002) 32 [arXiv:hep-ph/0202110]; H. Baer, C. Balazs, A. Belyaev, J. K. Mizukoshi, X. Tata and Y. Wang, JHEP 0207 (2002) 050 [arXiv:hep-ph/0205325]; R. Arnowitt and B. Dutta, arXiv:hep-ph/0211417.

[10] H. Baer, A. Mustafayev, S. Profumo, A. Belyaev and X. Tata, Phys. Rev. D 71 (2005) 095008 [arXiv:hep-ph/0412059]; H. Baer, A. Mustafayev, S. Profumo, A. Belyaev and X. Tata, JHEP 0507 (2005) 065, hep-ph/0504001; J. R. Ellis, K. A. Olive and P. Sandick, Phys. Rev. D 78 (2008) 075012 [arXiv:0805.2343 [hep-ph]]; J. Ellis, F. Luo, K. A. Olive and P. Sandick, Eur. Phys. J. C 73 (2013) 2403 [arXiv:1212.4476 [hep-ph]].

[11] J. Ellis, K. Olive and Y. Santoso, Phys. Lett. B 539 (2002) 107 [arXiv:hep-ph/0204192]; J. R. Ellis, T. Falk, K. A. Olive and Y. Santoso, Nucl. Phys. B 652 (2003) 259 [arXiv:hep-ph/0210205]. 
[12] See, for example, C. F. Berger, J. S. Gainer, J. L. Hewett and T. G. Rizzo, JHEP 0902, 023 (2009) [arXiv:0812.0980 [hep-ph]]; S. S. AbdusSalam, B. C. Allanach, F. Quevedo, F. Feroz and M. Hobson, Phys. Rev. D 81, 095012 (2010) [arXiv:0904.2548 [hep-ph]]; J. A. Conley, J. S. Gainer, J. L. Hewett, M. P. Le and T. G. Rizzo, Eur. Phys. J. C 71, 1697 (2011) [arXiv:1009.2539 [hep-ph]]; J. A. Conley, J. S. Gainer, J. L. Hewett, M. P. Le and T. G. Rizzo, [arXiv:1103.1697 [hep-ph]]; B. C. Allanach, A. J. Barr, A. Dafinca and C. Gwenlan, JHEP 1107, 104 (2011) [arXiv:1105.1024 [hep-ph]]; S. Sekmen, S. Kraml, J. Lykken, F. Moortgat, S. Padhi, L. Pape, M. Pierini and H. B. Prosper et al., JHEP 1202 (2012) 075 [arXiv:1109.5119 [hep-ph]]; A. Arbey, M. Battaglia and F. Mahmoudi, Eur. Phys. J. C 72 (2012) 1847 [arXiv:1110.3726 [hep-ph]]; A. Arbey, M. Battaglia, A. Djouadi and F. Mahmoudi, Phys. Lett. B 720 (2013) 153 [arXiv:1211.4004 [hep-ph]]; M. W. Cahill-Rowley, J. L. Hewett, A. Ismail and T. G. Rizzo, Phys. Rev. D 88 (2013) 3, 035002 [arXiv:1211.1981 [hep-ph]]; C. Strege, G. Bertone, G. J. Besjes, S. Caron, R. Ruiz de Austri, A. Strubig and R. Trotta, JHEP 1409 (2014) 081 [arXiv:1405.0622 [hep-ph]]; M. Cahill-Rowley, J. L. Hewett, A. Ismail and T. G. Rizzo, Phys. Rev. D 91 (2015) 5, 055002 [arXiv:1407.4130 [hep-ph]]; L. Roszkowski, E. M. Sessolo and A. J. Williams, JHEP 1502, 014 (2015) [arXiv:1411.5214 [hep-ph]]; M. E. C. Catalan, S. Ando, C. Weniger and F. Zandanel, arXiv:1503.00599 [hep-ph]; J. Chakrabortty, A. Choudhury and S. Mondal, arXiv:1503.08703 [hep-ph].

[13] F. Feroz and M. P. Hobson, Mon. Not. Roy. Astron. Soc. 384, 449 (2008) [arXiv:0704.3704 [astro-ph]].

[14] F. Feroz, M. P. Hobson and M. Bridges, Mon. Not. Roy. Astron. Soc. 398 (2009) 1601 [arXiv:0809.3437 [astro-ph]].

[15] F. Feroz, M. P. Hobson, E. Cameron and A. N. Pettitt, arXiv:1306.2144 [astro-ph.IM].

[16] G. Belanger, F. Boudjema, A. Pukhov and A. Semenov, Comput. Phys. Commun. 185 (2014) 960 [arXiv:1305.0237 [hep-ph]].

[17] We base our analysis on G. Aad et al. [ATLAS Collaboration], arXiv:1405.7875 [hep-ex]. Full ATLAS Run 1 results can be found at https://twiki.cern.ch/twiki/bin/view/ At lasPublic/SupersymmetryPublicResults. See also S. Chatrchyan et al. [CMS Collaboration], JHEP 1406 (2014) 055 [arXiv:1402.4770 [hep-ex]].

Full CMS Run 1 results can be found at https://twiki.cern.ch/twiki/bin/view/ CMSPublic/PhysicsResultsSUS.

[18] O. Buchmueller, M. Citron, J. Ellis, S. Guha, J. Marrouche, K. A. Olive, K. de Vries and J. Zheng, arXiv:1505.04702 [hep-ph]. 